OPEN ACCESS

Edited by:

Tianhui Chen,

Zhejiang Cancer Hospital, China

Reviewed by:

Yimin Zhu,

Zhejiang University, China Hamideh Salimzadeh,

Tehran University of Medical

Sciences, Iran

*Correspondence:

$\mathrm{Ni} \mathrm{Li}$

nli@cicams.ac.cn

Min Dai

daimin2002@hotmail.com

Jie $\mathrm{He}$

prof.jiehe@gmail.com

Specialty section:

This article was submitted to

Cancer Epidemiology and Prevention,

a section of the journal

Frontiers in Oncology

Received: 23 July 2019 Accepted: 26 September 2019 Published: 17 October 2019

Citation:

Li X, Chen H, Wang G, Feng X, Lyu Z, Wei L, Wen Y, Chen S, Wu S, Hang D, Dai M, Li N and He J (2019) Metabolic Syndrome Components and the Risk of Colorectal Cancer: A

Population-Based Prospective Study in Chinese Men. Front. Oncol. 9:1047.

doi: 10.3389/fonc.2019.01047

\section{Metabolic Syndrome Components and the Risk of Colorectal Cancer: A Population-Based Prospective Study in Chinese Men}

\author{
Xin Li ${ }^{1}$, Hongda Chen ${ }^{1}$, Gang Wang ${ }^{2}$, Xiaoshuang Feng ${ }^{1}$, Zhangyan Lyu ${ }^{1}$, Luopei Wei ${ }^{1}$, \\ Yan Wen ${ }^{1}$, Shuohua Chen ${ }^{3}$, Shouling $\mathrm{Wu}^{3}$, Dong Hang ${ }^{4}$, Min $\mathrm{Dai}^{{ }^{*}}$, $\mathrm{Ni} \mathrm{Li}^{1 *}$ and Jie $\mathrm{He}^{1,5 *}$ \\ 1 Office of Cancer Screening, National Cancer Center/National Clinical Research Center for Cancer/Cancer Hospital, Chinese \\ Academy of Medical Sciences and Peking Union Medical College, Beijing, China, ${ }^{2}$ Department of Oncology, Kailuan General \\ Hospital, Tangshan, China, ${ }^{3}$ Health Department of Kailuan (Group), Tangshan, China, ${ }^{4}$ Department of Epidemiology, School \\ of Public Health, Nanjing Medical University, Nanjing, China, ${ }^{5}$ Department of Thoracic Surgery, National Cancer \\ Center/National Clinical Research Center for Cancer/Cancer Hospital, Chinese Academy of Medical Sciences and Peking \\ Union Medical College, Beijing, China
}

Background: To investigate the association between metabolic syndrome (MetS) and the risk of colorectal cancer (CRC) in Chinese men, this study was performed based on data from a large prospective cohort study conducted in China named the Kailuan men cohort study.

Methods : A total of 104,333 eligible men who participated in biennial examinations at least once from 2006 to 2015 were recruited. Cox proportional hazards regression models were used to estimate the effects of MetS components on CRC risk.

Results: During an 824,211.96 person-years follow-up, 394 CRC cases were verified. Participants with high waist circumference ( $\geq 90 \mathrm{vs.}<90 \mathrm{~cm}$ ) had a significantly higher risk of developing incident $\mathrm{CRC}(\mathrm{HR}=1.32,95 \% \mathrm{Cl}$ : 1.07-1.64). Compared with participants with no MetS components, the HRs (95\% Cl) of developing CRC for men with 1, 2, and $\geq 3$ MetS components were 1.53 (1.01-2.32), 1.42 (0.94-2.14), and 1.70 (1.12-2.56), respectively. In addition, a statistically significant trend $(P$ for trend $=0.04)$ of increased CRC risk with an increasing number of abnormal MetS components was observed. Furthermore, compared with no MetS components, the combination of high waist circumference and elevated fasting blood glucose along with normal levels of the other 3 components, showed a 126\% increased risk of CRC.

Conclusions: Our study suggests that CRC risk is correlated with the number of abnormal MetS components in Chinese men. Men with high waist circumference and elevated fasting blood glucose may have a higher CRC risk even if they do not meet the MetS diagnostic criteria.

Keywords: colorectal cancer, metabolic syndrome, cohort studies, prospective study, Chinese men 


\section{INTRODUCTION}

Colorectal cancer (CRC) is the fourth most common incident cancer around the world; $10.2 \%$ of the estimated $1,849,518$ new CRC cases and $9.2 \%$ of the estimated 880,792 CRC-related deaths worldwide occurred in 2018 (1). In China, an estimated 376,300 new cases and 191,000 deaths from CRC occurred in 2015. Moreover, in recent decades, an increasing trend in the agestandardized incidence and mortality rates of CRC was observed among mainland Chinese men (2).

Metabolic syndrome (MetS) has been used to describe a composite condition characterized by a cluster of components, including high waist circumference (WC), elevated fasting blood glucose (FBG), elevated blood pressure (BP), elevated triglyceride (TG), and reduced high-density lipoprotein cholesterol (HDLC). The increasing prevalence of MetS is now both a major clinical problem and a common public health problem (3). Several studies have suggested that MetS was related to an increased risk of cardiovascular disease $(4,5)$, type 2 diabetes mellitus (T2DM) (6), and all-cause mortality (7). Moreover, epidemiologic evidence has also suggested that the MetS may play a significant role in cancer development $(8,9)$.

Since 2001, several studies have investigated the association between MetS and CRC risk but have shown inconsistent results (10-12), especially among the Asian population (13). Additionally, evidence based on large prospective population studies is lacking in China. Moreover, it is not clear whether the risk of CRC could differ based on different combinations of MetS components, which is crucial for the risk stratification of individuals regarding the development of CRC.

Thus, we performed the current study with a large prospective cohort of Chinese men with the aim of examining the associations of MetS and its components with the risk of CRC. Mostly, we focused on both single and combinations of MetS components, which may be helpful in developing feasible prevention strategies for CRC.

\section{METHODS}

\section{Study Design and Population}

The data were derived from a prospective dynamic cohort study of the Kailuan Group, which is a functional community in Tangshan City, Hebei Province in the northern region of China. Tangshan is located $\sim 90$ miles southeast of Beijing and is representative of the total population of China from a socioeconomic perspective (14). The Kailuan Group is a comprehensive company that manages machine manufacturing, the coal industry, coking, chemical production, transportation,

Abbreviations: CRC, colorectal cancer; MetS, metabolic syndrome; WC, waist circumference; FBG, fasting blood glucose; BP, blood pressure; TG, triglyceride; HDL-C, high-density lipoprotein cholesterol; T2DM, type 2 diabetes mellitus; GPO, glycerol phosphate oxidase; AHA/NHLBI, American Heart Association, and National Heart, Lung, and Blood Institute; ICD-10, International Classification of Diseases, tenth revision; CI, confidence interval; CNY, Chinese Yuan; ARIC, Atherosclerosis Risk in Communities; Me-Can, the metabolic syndrome and cancer project; ROS, reactive oxygen species; IGF, insulin-like growth factor; IGFBP, insulin-like growth factor-binding proteins; ATBC, Alpha-Tocopherol, Beta-Carotene Cancer Prevention. healthcare, education, etc. Since May 2006, over 130,000 individuals were included in the biennial questionnaire interview and clinical examination at eleven affiliated hospitals in the Kailuan Group (15). The rationale and methodology of the Kailuan study have been described previously (16).

Participants who met the following criteria were recruited for the study: (1) men aged $\geq 18$ years, (2) participants who provided informed consent and (3) individuals who completed the questionnaire interview. Participants without baseline information on WC $(n=3,786), \operatorname{FBG}(n=345)$, history of T2DM or treatment for T2DM $(n=427)$, BP $(n=135)$, history of or treatment for hypertension $(n=1,558)$, TG $(n=18)$, and HDL-C $(n=10)$ were excluded. Finally, a total of 104,333 participants were enrolled in the present study.

All participants provided written informed consent according to the guidelines of the Declaration of Helsinki, and the study was approved by the ethics committee of Kailuan General Hospital.

\section{Assessment of Exposure}

In each biennial survey cycle, face-to-face questionnaire interviews and health examinations were facilitated by welltrained physicians or nurses using a standardized protocol to gather information on demographics, socioeconomic characteristics, lifestyle, medical history, and laboratory tests at baseline. Smoking was defined as at least one cigarette weekly for over 6 months, and participants were classified as "never smokers," "former smokers," or "current smokers." The definition of alcohol drinking was the consumption of alcohol at least once per month for over 6 months, and participants were categorized as "never drinkers," "former drinkers," "<1 time per day," or " $\geq 1$ time per day." Sitting time was categorized as " $<4 \mathrm{~h}$ per day, " $4-8 \mathrm{~h}$ per day," and " $\geq 8 \mathrm{~h}$ per day." WC was measured horizontally at the midpoint between the anterior superior iliac crest and the inferior rib. Systolic BP and diastolic BP levels were taken on the left arm using a mercury sphygmomanometer with a cuff of suitable size after participants rested for $5 \mathrm{~min}$, which followed the standard recommended procedures. Two readings were taken at a 5-min interval, and the mean value of the two measurements was recorded for data analysis (17).

Morning fasting blood samples from each participant were examined using a standard operating procedure, and FBG levels were determined by the hexokinase/glucose-6-phosphate dehydrogenase method. The coefficient of variance of blind quality control samples was $<2.0 \%$. TG levels were detected by the glycerol phosphate oxidase (GPO) method (interassay coefficient of variance $<10 \%$ ), and HDL-C levels were detected in the supernatant after the sediment of apolipoprotein Bcontaining lipoproteins with dextran sulfate and magnesium chloride (15). All blood samples were measured using an autoanalyzer (Hitachi 747; Hitachi, Tokyo, Japan) (18).

\section{Definition of Metabolic Syndrome}

We defined MetS according to the American Heart Association, and National Heart, Lung, and Blood Institute (AHA/NHLBI) criteria in 2009 ( $\geq 3$ of the necessary 5 criteria): (a) high WC levels $(\geq 90 \mathrm{~cm}$ ), (b) elevated FBG levels (or antidiabetic medication for the treatment of previously elevated glucose) 
( $\geq 5.6 \mathrm{mmol} / \mathrm{L}$ ), (c) elevated BP levels (or antihypertensive medication treatment for previous hypertension) (systolic $\geq 130$ or diastolic $\geq 85 \mathrm{~mm} \mathrm{Hg}$ ), (d) elevated TG levels $(\geq 1.7 \mathrm{mmol} / \mathrm{L})$ and (e) reduced HDL-C levels $(<1.03 \mathrm{mmol} / \mathrm{L})(3)$.

\section{Assessment of Outcomes}

The endpoint was newly diagnosed cancer, death, or administrative censoring (December 31, 2015), whichever came first. For the duration of the follow-up, new cases were identified by tracking participants once they participated in regular questionnaires and health check-ups biennially. In addition, incident cancer cases were assessed annually by examining medical records linked with the Tangshan medical insurance system, and discharge summaries were also tracked from the eleven affiliated hospitals. Furthermore, death certificates from the Kailuan social security system were obtained to further confirm the outcome information (19).

The CRC cases were determined by study physicians' review of medical records. Information on pathological diagnoses, imaging diagnoses (including magnetic resonance imaging and computerized tomographic scanning), and blood biochemical examination were collected to assess incident CRC cases. Cancer was coded according to the International Classification of Diseases, tenth revision (ICD-10), and CRC was coded as C18-C20.

\section{Statistical Analysis}

Proportions and Chi-square tests were used to describe and compare categorical variables. The mean (standard deviation, SD) and $T$-test were used to describe and compare continuous variables. Person-years of risk was calculated from the date of recruitment until the date of first incident cancer, death, or end of follow-up (December 31, 2015), whichever occurred first. We used multivariable Cox proportional hazards regression models to estimate the association between MetS and MetS components with the risk of developing CRC. Model 1 was adjusted for age (continuous). Model 2 was additionally adjusted for income ( $<500$ Chinese Yuan (CNY) per month, 500-1,000 CNY per month, or $\geq 1,000 \mathrm{CNY}$ per month), education level (illiterate/primary school, junior high school, senior high school, or college and above), frequency of tobacco smoking (never smokers, former smokers, or current smokers), frequency of alcohol drinking (never drinkers, former drinkers, $<1$ time per day, or $\geq 1$ time per day), and sitting time ( $<4 \mathrm{~h}$ per day, $4-8 \mathrm{~h}$ per day, or $>8 \mathrm{~h}$ per day). Tests for linear trends across quartiles of MetS were performed by modeling the categories as continuous variables. To evaluate the potential effect of various combinations of MetS components, we conducted multivariable-adjusted risk of CRC development associated with combinations of MetS components, compared to participants with no components. Sensitivity analyses were performed to evaluate the consistency of our findings. First, we repeated the main analyses after excluding participants with a history of taking antidiabetic medication or a history of antihypertensive medication use; second, to examine the possibility of reverse causation, we excluded CRC cases that occurred during the first 3 years of follow-up.
All data management and statistical analyses were conducted using SAS, version 9.4 (SAS Institute Inc, Cary, NC, USA). The statistical tests were two-sided, and a $P$ value $<0.05$ was considered statistically significant.

\section{RESULTS}

\section{Baseline Characteristics of the Baseline Study Participants}

By December 2015, a total of 104,333 eligible men participants with a mean age of 51.2 years were recruited for this study. During a median follow-up of 8.9 years, 394 incident CRC cases were verified over a total of 824,211.96 person-years. The mean values of WC, FBG, DBP, SBP, TG, and HDL-C were $87.97 \mathrm{~cm}, 5.54 \mathrm{mmol} / \mathrm{L}, 84.64 \mathrm{mmHg}, 132.21 \mathrm{mmHg}, 1.72$ $\mathrm{mmol} / \mathrm{L}$, and $1.51 \mathrm{mmol} / \mathrm{L}$, respectively. As shown in Table 1, compared to participants without CRC, incident CRC cases were more likely to be older, have lower education levels (illiterate or primary school) and were more prone to alcohol drinking (all $P$ values $<0.05)$. The levels of WC $(P<0.001)$, FBG $(P=0.05)$, and SBP $(P<0.001)$ were higher among participants with CRC than among those without CRC. However, the levels of DBP $(P=0.68)$, TG $(P=0.99)$, and HDL-C $(P=0.16)$ were similar between the two groups (Table 1).

\section{Associations Between Individual Components of MetS and CRC Risk}

In multivariable-adjusted models, compared to the participants with normal WC $(<90 \mathrm{~cm})$, men with a high WC $(\geq 90 \mathrm{~cm})$ had a significantly higher risk of developing incident $\mathrm{CRC}(\mathrm{HR}=1.32$, 95\% CI: $1.07-1.64)$. Men with elevated FBG ( $\geq 5.6 \mathrm{mmol} / \mathrm{l}$ or antidiabetic medication for the treatment of previously elevated glucose vs. $<5.6 \mathrm{mmol} / 1)$ had an increased risk of CRC with borderline significance ( $\mathrm{HR}=1.19,95 \% \mathrm{CI}$ : 0.96-1.48). Men with elevated TG ( $\geq 1.7 \mathrm{mmol} / 1 \mathrm{vs.}<1.7 \mathrm{mmol} / 1)$ and reduced HDLC $(<1.03 \mathrm{mmol} / \mathrm{L}$ vs. $\geq 1.03 \mathrm{mmol} / \mathrm{L})$ had an increased but not statistically significant risk of CRC development ( $\mathrm{HR}=1.12,95 \%$ CI: $0.90-1.41 ; \mathrm{HR}=1.24,95 \% \mathrm{CI}$ : $0.84-1.84$, respectively). No increased risk was observed for men with elevated $\mathrm{BP}$ ( $\mathrm{SBP} \geq 130$ or DBP $\geq 85 \mathrm{~mm} \mathrm{Hg}$ or antihypertensive medication treatment for previous hypertension vs. normal) $(\mathrm{HR}=1.04,95 \% \mathrm{CI}$ : 0.82-1.31; Table 2).

\section{Association Between MetS and CRC Risk}

Multivariate analysis showed that compared to men with no MetS components, the HRs (95\% CI) for developing CRC among men with 1,2 , and $\geq 3$ MetS components were 1.53 (1.01$2.32), 1.42$ (0.94-2.14), and 1.70 (1.12-2.56), respectively, which showed a statistically significant trend $(P$ for trend $=0.04)$ of increased CRC risk with an increasing number of abnormal MetS components. In addition, when compared to those who did not meet the MetS diagnostic criteria (no. of MetS components $<3$ ), men with MetS had an increased risk of CRC with borderline significance $(\mathrm{HR}=1.22$, 95\% CI: 0.97-1.53; Table 3). 
TABLE 1 | Baseline characteristics of men by CRC, Kailuan cohort, 2006-2015.

\begin{tabular}{|c|c|c|c|c|}
\hline \multirow[t]{2}{*}{ Characteristics } & \multirow{2}{*}{$\begin{array}{l}\text { Total cohort } \\
(n=104,333)\end{array}$} & \multicolumn{2}{|c|}{ CRC } & \multirow[t]{2}{*}{$P$ value } \\
\hline & & Yes $(n=394)$ & No $(n=103,939)$ & \\
\hline $\mathrm{WC}(\mathrm{cm})^{\mathrm{a}}$ & 87.97 (9.68) & $89.94(9.86)$ & 87.96 (9.68) & $<0.001$ \\
\hline Age $\left(\right.$ years) ${ }^{\mathrm{a}}$ & $51.21(13.46)$ & $59.84(9.89)$ & $51.17(13.46)$ & $<0.001$ \\
\hline $\mathrm{FBG}(\mathrm{mmol} / \mathrm{L})^{\mathrm{a}}$ & $5.54(1.70)$ & $5.74(2.00)$ & $5.54(1.70)$ & 0.05 \\
\hline $\mathrm{DBP}(\mathrm{mmHg})^{\mathrm{a}}$ & $84.64(11.72)$ & $84.89(11.40)$ & 84.66 (11.73) & 0.68 \\
\hline $\operatorname{SBP}(m m H g)^{a}$ & $132.21(20.66)$ & $136.02(21.41)$ & $132.20(20.65)$ & $<0.001$ \\
\hline $\mathrm{TG}(\mathrm{mmol} / \mathrm{L})^{\mathrm{a}}$ & $1.72(1.53)$ & $1.72(1.22)$ & $1.72(1.53)$ & 0.99 \\
\hline HDL-C (mmol/L) $)^{a}$ & $1.51(0.45)$ & $1.54(0.44)$ & $1.51(0.45)$ & 0.16 \\
\hline \multicolumn{5}{|l|}{ Education level ${ }^{b}$} \\
\hline Illiterate or primary school & $11,978(11.46)$ & 75 (19.04) & $11,903(11.26)$ & $<0.0001$ \\
\hline Junior high school & 67,605 (66.75) & $265(67.26)$ & $69,305(66.74)$ & \\
\hline Senior high school & 14,524 (13.93) & $38(9.64)$ & 14,486 (13.95) & \\
\hline College and above & $8,158(7.83)$ & $16(4.06)$ & $8,142(7.84)$ & \\
\hline \multicolumn{5}{|l|}{ Income (yuan per month) ${ }^{b}$} \\
\hline$<500$ & $25,186(25.12)$ & $88(23.10)$ & $25,098(25.13)$ & 0.006 \\
\hline $500-1,000$ & $55,668(55.52)$ & $240(62.99)$ & $55,428(55.49)$ & \\
\hline$\geq 1,000$ & $19,409(19.36)$ & $53(13.91)$ & 19,356 (19.38) & \\
\hline \multicolumn{5}{|l|}{ Smoking status } \\
\hline Never & 54,435 (56.43) & $199(56.06)$ & $54,236(56.43)$ & 0.35 \\
\hline Former & $3,986(4.13)$ & $20(5.63)$ & 3,966 (4.13) & \\
\hline Current & 38,052 (39.44) & $136(38.31)$ & 37,916 (39.45) & \\
\hline \multicolumn{5}{|l|}{ Alcohol drinking status ${ }^{b}$} \\
\hline Never & $52,228(50.07)$ & $192(48.73)$ & $52,036(50.08)$ & $<0.0001$ \\
\hline Former & 4,462 (4.28) & $18(4.57)$ & $4,444(4.28)$ & \\
\hline$<1$ time/day & $25,790(24.73)$ & $65(16.50)$ & 25,273 (24.76) & \\
\hline$\geq 1$ time/day & 21,823 (20.92) & 119 (30.20) & 21,704 (20.89) & \\
\hline \multicolumn{5}{|l|}{ Sitting time $(\mathrm{h} / \text { day })^{\mathrm{b}}$} \\
\hline$<4$ & 72,543 (72.27) & $293(74.74)$ & 72,250 (72.26) & 0.07 \\
\hline $4-8$ & 24,526 (24.43) & 94 (23.98) & $24,432(24.44)$ & \\
\hline$\geq 8$ & 3,310 (3.30) & $5(1.28)$ & 3,305 (3.31) & \\
\hline
\end{tabular}

CRC, colorectal cancer; WC, waist circumference; FBG, fasting blood glucose; BP, blood pressure; TG, triglyceride; HDL-C, high-density lipoprotein cholesterol.

${ }^{a}$ Mean (standard deviation), $P$ values from $T$-test.

${ }^{b} N(\%), P$ values from the $\chi^{2}$ test.

\section{Combined Associations of MetS Components With CRC Risk}

We examined the HRs of all combinations (excluding 4 combinations owing to the limited number of cases) of the $5 \mathrm{MetS}$ components. The no MetS component group was used as the reference category, and a combination of high WC and elevated FBG with normal levels of the other 3 components was associated with an increased risk for CRC (HR $=2.26,95 \%$ CI: 1.10-4.65). In addition, the combination of high WC, elevated FBG and elevated BP ( $\mathrm{HR}=1.82,95 \% \mathrm{CI}: 1.08-3.08)$; the combination of high WC, elevated FBG and elevated TG ( $\mathrm{HR}=2.66,95 \% \mathrm{CI}$ : 1.21-5.81); and the combination of high WC, elevated BP and reduced HDL-C (HR $=2.75,95 \%$ CI: $1.06-7.12)$ were associated with a higher risk of developing CRC (Table 4).

\section{Sensitivity Analysis}

In the sensitivity analysis, when excluding individuals with antidiabetic medication use history $(n=2,578$, case no.
$=19)$ and individuals with antihypertensive medication use history $(n=10,770$, case no. $=59)$, the men with $\geq 3$ MetS components $(\mathrm{HR}=1.64,95 \% \mathrm{CI}: 1.08-2.49, \mathrm{HR}=1.64,95 \%$ CI: 1.07-2.51) still had a significantly increased risk of CRC development. After excluding CRC cases (no.=99) that occurred during the first 3 years of follow-up, the results were similar $\left(\mathrm{HR}_{3}\right.$ components vs. 0 components $=1.54,95 \%$ CI: $0.96-2.49$; Table 5). The results of the sensitivity analyses did not alter the main findings.

\section{DISCUSSION}

Based on this large prospective cohort study among Chinese men, we documented that high WC was associated with CRC development. When considered combinedly, the number of abnormal MetS components was linearly associated with increased CRC risk. Notably, among MetS components, the combination of high WC and elevated FBG may be associated 
TABLE 2 | Associations between individual components of MetS and CRC risk in men, Kailuan cohort, 2006-2015.

\begin{tabular}{|c|c|c|c|c|}
\hline \multirow[t]{2}{*}{ Groups } & \multirow[t]{2}{*}{ Cases } & \multirow[t]{2}{*}{ Person-years } & \multicolumn{2}{|c|}{ HR (95\% Cl) } \\
\hline & & & Model $1^{a}$ & Model $2^{b}$ \\
\hline \multicolumn{5}{|l|}{ WC (cm) } \\
\hline$<90$ & 190 & $479,112.87$ & ref & ref \\
\hline$\geq 90$ & 204 & $345,099.09$ & 1.27 (1.05-1.55) & $1.32(1.07-1.64)$ \\
\hline \multicolumn{5}{|c|}{ FBG (mmol/l)c } \\
\hline Normal & 242 & $562,176.91$ & ref & ref \\
\hline High & 152 & $262,035.04$ & $1.26(1.03-1.55)$ & $1.19(0.96-1.48)$ \\
\hline \multicolumn{5}{|c|}{$B P(m m H g)^{d}$} \\
\hline Normal & 123 & $333,917.25$ & ref & ref \\
\hline High & 271 & $490,294.71$ & $1.05(0.85-1.31)$ & $1.04(0.82-1.31)$ \\
\hline \multicolumn{5}{|c|}{ TG (mmol/l) } \\
\hline$<1.7$ & 259 & $553,472.89$ & ref & ref \\
\hline$\geq 1.7$ & 135 & $270,739.07$ & $1.18(0.96-1.46)$ & $1.12(0.90-1.41)$ \\
\hline \multicolumn{5}{|c|}{ HDL-C (mmol/l) } \\
\hline$<1.03$ & 33 & $59,545.17$ & $1.26(0.88-1.80)$ & $1.24(0.84-1.84)$ \\
\hline$\geq 1.03$ & 361 & $764,666.79$ & ref & ref \\
\hline
\end{tabular}

CRC, colorectal cancer; MetS, metabolic syndrome; WC, waist circumference; FBG, fasting blood glucose; BP, blood pressure; TG, triglyceride; HDL-C, high-density lipoprotein cholesterol.

${ }^{\text {a }}$ Adjusted for age.

${ }^{b}$ Adjusted for age, education level, income status, frequency of tobacco smoking, frequency of alcohol drinking, and sitting time.

${ }^{c}$ High was defined as FBG levels $\geq 5.6 \mathrm{mmol} / \mathrm{L}$ or anti-diabetic medication for the treatment of previously elevated glucose. Normal was defined as $F B G<5.6 \mathrm{mmol} / \mathrm{L}$.

${ }^{d}$ High was defined as systolic blood pressure $\geq 130$ or diastolic blood pressure $\geq 85 \mathrm{~mm}$ $\mathrm{Hg}$ or antihypertensive medication treatment for previously hypertension, Normal was defined as systolic blood pressure $<130$ and diastolic blood pressure $<85 \mathrm{~mm} \mathrm{Hg}$.

with an increased CRC risk, even in individuals who did not meet the MetS diagnostic criteria. To our knowledge, this is the first large prospective cohort study to report the associations of both single and combinations of components of MetS with CRC risk among men in China, and the results could provide powerful evidence supporting a potential effect of MetS components on increased CRC risk.

The evidence of the associations between individual components of MetS and CRC risk differed as follows. The increased risk of CRC in men with high WC in this study was similar to that observed in previous studies $(10,20,21)$. The prospective cohort study Atherosclerosis Risk in Communities (ARIC) in the United States showed that compared with men with a WC $<102 \mathrm{~cm}$, those with abdominal obesity (WC $>102 \mathrm{~cm})$ had a higher risk of CRC (RR $=1.40$; 95\% CI, 1.001.90) (10). A meta-analysis of seven prospective cohort studies in Europe revealed that WC was associated with the development of CRC among men (per 1-s.d. increment: $\mathrm{HR}=1.21,95 \%$ CI:1.08-1.35) (20).

The Korean Metabolic Syndrome Research Initiative study involving 175,677 participants with a mean follow-up of 4.7 years found that elevated FBG was associated with higher CRC risk in men ( $\mathrm{HR}=1.51,95 \% \mathrm{CI}: 1.12-2.05)$ (22). However, the Metabolic Syndrome and Cancer Project (Me-Can) of 289,866 adult men in Europe with a mean follow-up of 12.8 years showed
TABLE 3 | Associations between MetS and CRC risk in men, Kailuan cohort, 2006-2015.

\begin{tabular}{|c|c|c|c|c|}
\hline \multirow[t]{2}{*}{ Groups } & \multirow[t]{2}{*}{ Cases } & \multirow[t]{2}{*}{ Person-years } & \multicolumn{2}{|c|}{ HR $(95 \% \mathrm{Cl})$} \\
\hline & & & Model $1^{a}$ & Model $2^{b}$ \\
\hline \multicolumn{5}{|c|}{ No. of MetS components ${ }^{c}$} \\
\hline 0 & 33 & $131,781.60$ & ref & ref \\
\hline 1 & 112 & $237,461.25$ & $1.48(1.00-2.19)$ & $1.53(1.01-2.32)$ \\
\hline 2 & 118 & $240,331.66$ & $1.41(0.96-2.08)$ & $1.42(0.94-2.14)$ \\
\hline$\geq 3$ & 131 & $214,637.45$ & $1.74(1.18-2.55)$ & $1.70(1.12-2.56)$ \\
\hline$P$ for trend & & & 0.01 & 0.04 \\
\hline \multicolumn{5}{|c|}{ Dichotomously defined } \\
\hline No MetS & 263 & $609,574.51$ & ref & ref \\
\hline MetS & 131 & $214,637.45$ & $1.27(1.03-1.56)$ & $1.22(0.97-1.53)$ \\
\hline
\end{tabular}

CRC, colorectal cancer; MetS, metabolic syndrome; No, number.

a Adjusted for age.

${ }^{b}$ Adjusted for age, education level, income status, frequency of cigarette smoking, frequency of alcohol drinking, and sitting time.

${ }^{c}$ Cut-off points were based on the American Heart Association, and National Heart, Lung, and Blood Institute (AHA/NHLBI) criteria in 2009 ( $\geq 3$ of the necessary 5 criteria): (a) high WC levels ( $\geq 90 \mathrm{~cm}$ ), (b) elevated FBG levels (or antidiabetic medication for the treatment of previously elevated glucose) ( $\geq 5.6 \mathrm{mmol} / \mathrm{L}$ ), (c) elevated BP levels (or antihypertensive medication treatment for previous hypertension) (systolic $\geq 130$ or diastolic $\geq 85 \mathrm{~mm} \mathrm{Hg}$ ), (d) elevated TG levels ( $\geq 1.7 \mathrm{mmol} / \mathrm{L})$, (e) reduced HDL-C levels ( $<1.03 \mathrm{mmol} / \mathrm{L})$. MetS was defined as the presence of $\geq 3$ of the necessary 5 criteria.

no statistically significant association between CRC risk and FBG level in men (23). In most previous studies, hypertension has been related to a non-significant increased risk of CRC incidence $(11,24)$. Consistent with a cohort study in Austria (25) and the Alpha-Tocopherol, Beta-Carotene Cancer Prevention (ATBC) Study cohort (26), we found no significant association between CRC and elevated TG or reduced HDL-C.

Our observation of the association of CRC risk with MetS is consistent with the results of previous studies (10-12, 27-30). A meta-analysis of 15 datasets of men found a significantly positive association between MetS and CRC ( $\mathrm{RR}=1.33$, 95\% CI: $1.18-$ 1.50) as compared with those without MetS (13). Moreover, in 2006, the ARIC multicenter prospective cohort study involving 14,109 men reported a relationship of MetS based on high WC, elevated TG, reduced HDL-C, elevated BP and elevated FBG with CRC risk ( 3 vs. 0 components, $\mathrm{RR}=1.78,95 \% \mathrm{CI}$ : $1.0-3.6$; $\geq 3$ components vs. $<3$ components, $\mathrm{RR}=1.31,95 \% \mathrm{CI}: 0.9-1.9$ ) (10). The Physicians' Health Study also found that among men, each additional metabolic abnormality increased the risk of CRC $(\mathrm{RR}=1.16,95 \% \mathrm{CI}: 1.05-1.29)$ (11). Taken together, the results of the present study could be reasonable.

Several potential mechanisms might explain the associations between MetS components and the development of CRC (31). First, it has been proposed that MetS is represented by insulin resistance and hyperinsulinemia (32), and insulin resistance could stimulate the production of reactive oxygen species (ROS), which may damage DNA and contribute to malignant transformation (33). In addition, hyperinsulinemia leads to decreased hepatic synthesis of insulin-like growth factor (IGF)binding protein 1 (IGFBP-1) and protein 2 (IGFBP-2) and may result in increased bioactivity of IGF-I $(34,35)$, which directly induces the development of CRC due to its mitogenic 
TABLE 4 | Associations of MetS component combinations with CRC risk in men, Kailuan cohort, 2006-2015.

\begin{tabular}{|c|c|c|c|c|c|c|}
\hline \multicolumn{3}{|c|}{ Combination of components } & \multirow[t]{2}{*}{ Cases } & \multirow[t]{2}{*}{ Person-years } & \multicolumn{2}{|c|}{ HR (95\% Cl) } \\
\hline & & & & & Model $1^{a}$ & Model $2^{b}$ \\
\hline \multicolumn{3}{|l|}{ Null } & 33 & $131,781.60$ & ref & ref \\
\hline $\mathrm{WC} \uparrow$ & \multicolumn{2}{|l|}{$\mathrm{FBG} \uparrow$} & 10 & $15,809.74$ & $1.96(0.97-4.01)$ & $2.26(1.10-4.65)$ \\
\hline $\mathrm{WC} \uparrow$ & \multicolumn{2}{|l|}{$\mathrm{BP} \uparrow$} & 47 & $78,214.68$ & $1.47(0.93-2.31)$ & $1.50(0.93-2.43)$ \\
\hline$W C \uparrow$ & \multicolumn{2}{|l|}{$\mathrm{TG} \uparrow$} & 8 & $23,375.18$ & $1.32(0.61-2.87)$ & $1.51(0.69-3.32)$ \\
\hline $\mathrm{WC} \uparrow$ & \multicolumn{2}{|l|}{$\mathrm{HDL}-\mathrm{C} \downarrow$} & 1 & $4,477.33$ & $0.82(0.11-6.01)$ & $0.97(0.13-7.15)$ \\
\hline $\mathrm{FBG} \uparrow$ & \multicolumn{2}{|l|}{$\mathrm{BP} \uparrow$} & 23 & $51,737.01$ & $1.22(0.72-2.09)$ & $1.08(0.60-1.95)$ \\
\hline $\mathrm{FBG} \uparrow$ & \multicolumn{2}{|l|}{$\mathrm{TG} \uparrow$} & 5 & $12,748.92$ & $1.57(0.61-4.03)$ & $1.42(0.50-4.04)$ \\
\hline $\mathrm{FBG} \uparrow$ & \multicolumn{2}{|l|}{$\mathrm{HDL}-\mathrm{C} \downarrow$} & 0 & - & - & - \\
\hline $\mathrm{BP} \uparrow$ & \multicolumn{2}{|l|}{$\mathrm{TG} \uparrow$} & 20 & $42,342.43$ & $1.56(0.90-2.73)$ & $1.45(0.80-2.64)$ \\
\hline $\mathrm{BP} \uparrow$ & \multicolumn{2}{|l|}{$\mathrm{HDL}-\mathrm{C} \downarrow$} & 3 & $6,246.89$ & $1.32(0.40-4.32)$ & $1.56(0.47-5.13)$ \\
\hline $\mathrm{TG} \uparrow$ & \multicolumn{2}{|l|}{$\mathrm{HDL}-\mathrm{C} \downarrow$} & 1 & $3,171.50$ & $1.59(0.22-11.61)$ & $1.88(0.26-13.81)$ \\
\hline$W C \uparrow$ & $\mathrm{FBG} \uparrow$ & $\mathrm{BP} \uparrow$ & 33 & $43,628.16$ & $1.85(1.13-3.01)$ & $1.82(1.08-3.08)$ \\
\hline WC个 & $\mathrm{FBG} \uparrow$ & $\mathrm{TG} \uparrow$ & 9 & $12,425.13$ & $2.63(1.26-5.50)$ & $2.66(1.21-5.81)$ \\
\hline$W C \uparrow$ & $\mathrm{FBG} \uparrow$ & $\mathrm{HDL}-\mathrm{C} \downarrow$ & 1 & $1,107.33$ & - & - \\
\hline$W C \uparrow$ & $\mathrm{BP} \uparrow$ & $\mathrm{TG} \uparrow$ & 22 & $53,784.33$ & $1.20(0.70-2.06)$ & $1.13(0.62-2.04)$ \\
\hline$W C \uparrow$ & $\mathrm{BP} \uparrow$ & $\mathrm{HDL}-\mathrm{C} \downarrow$ & 5 & $6,054.71$ & $2.21(0.86-5.68)$ & $2.75(1.06-7.12)$ \\
\hline$W C \uparrow$ & $\mathrm{TG} \uparrow$ & HDL-C $\downarrow$ & 1 & $3,238.83$ & 1.33 (0.18-9.70) & $1.61(0.22-11.83)$ \\
\hline $\mathrm{FBG} \uparrow$ & $\mathrm{BP} \uparrow$ & $\mathrm{TG} \uparrow$ & 12 & $26,834.86$ & $1.43(0.74-2.78)$ & $1.32(0.64-2.72)$ \\
\hline $\mathrm{FBG} \uparrow$ & $\mathrm{BP} \uparrow$ & HDL-C $\downarrow$ & 1 & $2,192.96$ & $1.22(0.17-8.93)$ & $1.52(0.21-11.18)$ \\
\hline $\mathrm{FBG} \uparrow$ & $\mathrm{TG} \uparrow$ & $\mathrm{HDL}-\mathrm{C} \downarrow$ & 0 & - & - & - \\
\hline $\mathrm{BP} \uparrow$ & $\mathrm{TG} \uparrow$ & HDL-C $\downarrow$ & 0 & - & - & - \\
\hline \multicolumn{3}{|l|}{ All of 5} & 7 & 4625.48 & 4.41 (1.95-9.98) & $4.55(1.88-11.00)$ \\
\hline
\end{tabular}

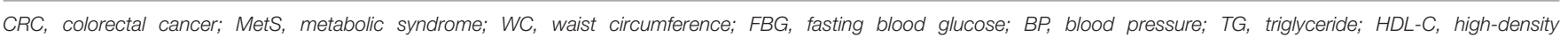
lipoprotein cholesterol.

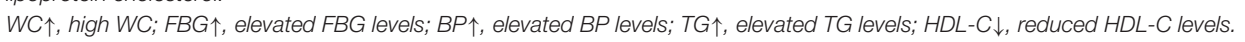

${ }^{a}$ Adjusted for age.

${ }^{b}$ Adjusted for age, education level, income status, frequency of cigarette smoking, frequency of alcohol drinking, and sitting time.

and antiapoptotic actions (33). Moreover, obese people have downregulated expression of adiponectin, an adipokine with anti-inflammatory and insulin-sensitizing properties, which can play an etiologic role in mutagenesis and may contribute to the development of cancer $(33,36)$. However, some evidence has identified obesity-related inflammation as a factor involved in cancer development, but more research is needed to confirm the extent of the association of factors that may be mediated by MetS $(37,38)$. Hence, more research is needed to better elucidate the potential biological mechanism underlying the relationship between MetS components and CRC.

In our study, the combined effects of the $5 \mathrm{MetS}$ components on the risk of CRC development varied; in particular, the coexistence of high WC and elevated FBG exhibited a significant association with higher CRC risk. The different associations of the combinations of the MetS components with CRC risk highlight the role of the combination of high WC and elevated FBG. This finding might have key clinical and scientific significance, as it indicates that individuals who have two components of MetS, such as high WC and elevated FBG, should receive attention, even when they do not meet the MetS diagnostic criteria. In addition, the combination of high WC, elevated BP and reduced HDL-C was associated with a significantly higher risk of developing CRC; however, the result may be unstable because of the small number of cases.
Our study has several strengths. One of the main strengths is its prospective design and the large sample size, which resulted in sufficient statistical power to detect modest differences and minimized the underlying bias due to preclinical disease. In addition, the laboratory tests (e.g., TG, HDL-C, and FBG) were conducted on the basis of standard procedures, which minimized the detection bias. Moreover, the WC and BP measurements were obtained by trained physicians or nurses rather than selfreport, avoiding misclassification bias. However, there are some limitations that should be considered when interpreting the results. The relatively short follow-up time (median, 8.9 years) is the major limitation of our study and precluded subtype analysis of CRC because of a limited number of cases. Additionally, in some groups, the small number of cases may have led to unstable results, and due to the limited number of cases, the four combinations of MetS components were not assessed. However, the Kailuan cohort is an ongoing dynamic cohort study; further follow-up should be conducted to identify more cases, and more stable results can be expected.

In conclusion, our study suggests that CRC risk is correlated with the number of abnormal MetS components in Chinese men. In addition, the combination of high WC and elevated FBG may have both clinical and public health significance for identifying individuals with high CRC risk. Therefore, the control of MetS, especially maintaining WC and FBG within an appropriate range, 
TABLE 5 | Sensitivity analysis of the association between MetS and CRC risk in men, Kailuan cohort, 2006-2015.

\begin{tabular}{|c|c|c|c|c|}
\hline \multirow{2}{*}{$\begin{array}{l}\text { No. of MetS } \\
\text { components }\end{array}$} & \multirow{2}{*}{ Cases } & \multirow[t]{2}{*}{ Person-years } & \multicolumn{2}{|c|}{ HR $(95 \% \mathrm{Cl})$} \\
\hline & & & Model $1^{a}$ & Model $2^{b}$ \\
\hline \multicolumn{5}{|c|}{ Excluding men with antidiabetic medication use history } \\
\hline 0 & 33 & $131,639.02$ & ref & ref \\
\hline 1 & 108 & $235,750.65$ & $1.44(0.97-2.13)$ & $1.52(1.00-2.29)$ \\
\hline 2 & 115 & $235,371.00$ & $1.41(0.96-2.08)$ & $1.44(0.95-2.17)$ \\
\hline$\geq 3$ & 119 & $202,361.22$ & $1.70(1.16-2.51)$ & $1.64(1.08-2.49)$ \\
\hline$P$ for trend & & & 0.01 & 0.06 \\
\hline \multicolumn{5}{|c|}{ Excluding men with antihypertensive medication use history } \\
\hline 0 & 33 & $131,781.60$ & ref & ref \\
\hline 1 & 107 & $223,255.16$ & $1.55(1.05-2.29)$ & $1.59(1.05-2.42)$ \\
\hline 2 & 97 & $212,505.33$ & $1.36(0.92-2.02)$ & $1.35(0.89-2.07)$ \\
\hline$\geq 3$ & 98 & $172,899.43$ & $1.69(1.14-2.51)$ & $1.64(1.07-2.51)$ \\
\hline$P$ for trend & & & 0.05 & 0.12 \\
\hline \multicolumn{5}{|c|}{ Excluding men who developed CRC within the first 3 years of follow-up } \\
\hline 0 & 25 & $131,590.14$ & ref & ref \\
\hline 1 & 86 & $236,940.80$ & $1.52(0.97-2.37)$ & $1.57(0.97-2.52)$ \\
\hline 2 & 92 & $239,801.29$ & $1.47(0.94-2.29)$ & $1.48(0.92-2.37)$ \\
\hline$\geq 3$ & 92 & $214,219.48$ & $1.64(1.05-2.55)$ & $1.54(0.96-2.49)$ \\
\hline$P$ for trend & & & 0.08 & 0.24 \\
\hline
\end{tabular}

CRC, colorectal cancer; MetS, metabolic syndrome; No, number.

aAdjusted for age.

${ }^{b}$ Adjusted for age, education level, income status, frequency of cigarette smoking, frequency of alcohol drinking, and sitting time.

may be a meaningful primary prevention strategy to decrease CRC risk among Chinese men.

\section{DATA AVAILABILITY STATEMENT}

The datasets for this manuscript are not publicly available because all our data are under regulation of both the National Cancer Center of China and Kailuan Group. Requests to access

\section{REFERENCES}

1. GLOBOCAN 2018. Available online at: http://gco.iarc.fr/today/data/ factsheets/cancers/10_8_9-Colorectum-fact-sheet.pdf (2019) (accessed 28 March 2019).

2. Chen W, Zheng R, Baade PD, Zhang S, Zeng H, Bray F, et al. Cancer statistics in China, 2015. CA Cancer J Clin. (2016) 66:115-32. doi: 10.3322/caac.21338

3. Alberti KGMM, Eckel RH, Grundy SM, Zimmet PZ, Cleeman JI, Donato KA, et al. Harmonizing the metabolic syndrome. Circulation. (2009) 120:1640-5. doi: 10.1161/CIRCULATIONAHA.109.192644

4. Grundy SM. Metabolic syndrome: connecting and reconciling cardiovascular and diabetes worlds. J Am Coll Cardiol. (2006) 47:1093-100. doi: 10.1016/j.jacc.2005.11.046

5. Mottillo S, Filion KB, Genest J, Joseph L, Pilote L, Poirier P, et al. The metabolic syndrome and cardiovascular risk a systematic review and meta-analysis. J Am Coll Cardiol. (2010) 56:1113-32. doi: 10.1016/j.jacc.2010.05.034

6. Grundy SM. Metabolic syndrome pandemic. Arterioscler Thromb Vasc Biol. (2008) 28:629-36. doi: 10.1161/ATVBAHA.107.151092

7. Katzmarzyk PT, Church TS, Blair SN. Cardiorespiratory fitness attenuates the effects of the metabolic syndrome on all-cause and cardiovascular the datasets should be directed to JH, hejie@cicams.ac.cn and SW, drwusl@163.com.

\section{ETHICS STATEMENT}

The studies involving human participants were reviewed and approved by The ethic committee of the Kailuan General Hospital. The patients/participants provided their written informed consent to participate in this study.

\section{AUTHOR CONTRIBUTIONS}

XL, NL, GW, SW, MD, and JH: conception and design. NL, MD, and JH: development of methodology and study supervision. XL, HC, XF, SC, LW, ZL, and YW: acquisition of data. XL, HC, and $\mathrm{DH}$ : analysis and interpretation of data. $\mathrm{XL}, \mathrm{NL}, \mathrm{MD}$, and $\mathrm{JH}$ : drafting, review, and/or revision of the study. All authors approved the final version of the study.

\section{FUNDING}

This work was supported by the National Key R\&D Program of China (grant numbers 2018YFC1315000/2018YFC1315001, 2016YFC1302500/2016YFC1302503), CAMS Innovation Fund for Medical Sciences (grant numbers 2017-I2M-1-006, 2019I2M-2-002), the Training Programme Foundation for the Talents in Beijing City (grant number 2017000021223TD05), Beijing Municipal Science and Technology Project (grant number D171100002617001), the and National Natural Science Foundation of China (grant number 81673265).

\section{ACKNOWLEDGMENTS}

The authors thank all the participants in the study and the members of the survey team from the Kailuan Cohort Study for their painstaking efforts to conduct the baseline survey and follow-up.

disease mortality in men. Arch Intern Med. (2004) 164:1092-7. doi: 10.1001/archinte.164.10.1092

8. Esposito K, Chiodini P, Colao A, Lenzi A, Giugliano D. Metabolic syndrome and risk of cancer: a systematic review and meta-analysis. Diabetes Care. (2012) 35:2402-11. doi: 10.2337/dc12-0336

9. Uzunlulu $\mathrm{M}$, Telci Caklili O, Oguz A. Association between metabolic syndrome and cancer. Ann Nutr Metab. (2016) 68:173-9. doi: 10.1159/000443743

10. Ahmed RL, Schmitz KH, Anderson KE, Rosamond WD, Folsom AR. The metabolic syndrome and risk of incident colorectal cancer. Cancer. (2006) 107:28-36. doi: 10.1002/cncr.21950

11. Sturmer T, Buring JE, Lee IM, Gaziano JM, Glynn RJ. Metabolic abnormalities and risk for colorectal cancer in the physicians' health study. Cancer Epidemiol Biomark Prevent. (2006) 15:2391-7. doi: 10.1158/1055-9965.EPI-06-0391

12. Antonio R, Mariangela A, Luigi B. Metabolic syndrome and cancer risk. Eur J Cancer. (2008) 44:293-7. doi: 10.1016/j.ejca.2007.11.005

13. Esposito K, Chiodini P, Capuano A, Bellastella G, Maiorino MI, Rafaniello $\mathrm{C}$, et al.Colorectal cancer association with metabolic syndrome and its components: a systematic review with meta-analysis. Endocrine. (2013) 44:634-47. doi: 10.1007/s12020-013-9939-5 
14. Hao X, Jianli W, Jinhong H, Hang Z, Jingsheng G, Shuohua C, et al. Association of ideal cardiovascular metrics and serum high-sensitivity Creactive protein in hypertensive population. PLoS ONE. (2013) 8:e81597. doi: 10.1371/journal.pone.0081597

15. Lyu Z, Li N, Wang G, Feng X, Chen S, Su K, et al. Independent and joint associations of blood lipids and lipoproteins with lung cancer risk in Chinese males: a prospective cohort study. Int J Cancer. (2019) 144:2972-84. doi: 10.1002/ijc.32051

16. Feng $\mathrm{X}$, Wang G, Li N, Lyu Z, Chen S, Wei L, et al. The association between fasting blood glucose and the risk of primary liver cancer in Chinese males: a population-based prospective study. Br J Cancer. (2017) 117:1405-11. doi: 10.1038/bjc.2017.296

17. Wang L, Cui L, Wang Y, Vaidya A, Chen S, Zhang C, et al. Resting heart rate and the risk of developing impaired fasting glucose and diabetes: the Kailuan prospective study[J]. Int J Epidemiol. (2015) 44:689-99. doi: 10.1093/ije/dyv079

18. Wang F, Wu S, Song Y, Tang X, Marshall R, Liang M, et al. Waist circumference, body mass index and waist to hip ratio for prediction of the metabolic syndrome in Chinese[J]. NMCD. (2009) 19:542-47. doi: 10.1016/j.numecd.2008.11.006

19. Wei L, Li N, Wang G, Feng X, Lyu Z, Li X, et al. Waist circumference might be a predictor of primary liver cancer: a population-based cohort study. Front Oncol. (2018) 8:607. doi: 10.3389/fonc.2018.00607

20. Freisling H, Arnold M, Soerjomataram I, O’Doherty MG, Ordonez-Mena JM, Bamia C, et al. Comparison of general obesity and measures of body fat distribution in older adults in relation to cancer risk: meta-analysis of individual participant data of seven prospective cohorts in Europe. $\mathrm{Br} \mathrm{J}$ Cancer. (2017) 116:1486-97. doi: 10.1038/bjc.2017.106

21. Calle EE, Rudolf K. Overweight, obesity and cancer: epidemiological evidence and proposed mechanisms. Nat Rev Cancer. (2004) 4:579-91. doi: $10.1038 / \mathrm{nrc1} 1408$

22. Shin $\mathrm{H}$ Y, Jung K J, Linton J A, Jee SH. Association between fasting serum glucose levels and incidence of colorectal cancer in Korean men: The Korean Cancer Prevention Study-II[J]. Metabolism. (2014) 63:1250-6. doi: 10.1016/j.metabol.2014.07.006

23. Stocks T, Lukanova A, Bjørge T, Ulmer H, Manjer J, Almquist M, et al. Metabolic factors and the risk of colorectal cancer in 580,000 men and women in the metabolic syndrome and cancer project. (Me-Can). Cancer. (2011) 117:2398-407. doi: 10.1002/cncr.25772

24. Rosengren A, Himmelmann A, Wilhelmsen L, Branehog I, Wedel H. Hypertension and long-term cancer incidence and mortality among Swedish men. J Hypertens. (1998) 16:933-40. doi: 10.1097/00004872-199816070-00006

25. Ulmer H, Borena W, Rapp K, Klenk J, Strasak A, Diem G, et al. Serum triglyceride concentrations and cancer risk in a large cohort study in Austria. Br J Cancer. (2009) 101:1202-6. doi: 10.1038/sj.bjc.6605264

26. Jiyoung A, Unhee L, Weinstein SJ, Arthur S, Hayes RB, Jarmo V, et al. Prediagnostic total and high-density lipoprotein cholesterol and risk of cancer. Cancer Epidemiol Biomark Prev. (2009) 18:2814-21. doi: 10.1158/1055-9965.EPI-08-1248

27. Inoue $M$, Noda $M$, Kurahashi $N$, Iwasaki M, Sasazuki S, Iso H, et al. Impact of metabolic factors on subsequent cancer risk: results from a large-scale population-based cohort study in Japan. Eur J Cancer Prev. (2009) 18:240-7. doi: 10.1097/CEJ.0b013e3283240460

28. Choi YJ, Lee DH, Han KD, Shin CM, Kim N. Abdominal obesity, glucose intolerance and decreased high-density lipoprotein cholesterol as components of the metabolic syndrome are associated with the development of colorectal cancer. Eur J Epidemiol. (2018) 33:1077-85. doi: 10.1007/s10654-01 8-0440-6

29. Aleksandrova K, Boeing H, Jenab M, Bas Bueno-de-Mesquita $H$, Jansen E, van Duijnhoven FJ, et al. Metabolic syndrome and risks of colon and rectal cancer: the European prospective investigation into cancer and nutrition study. Cancer Prev Res. (2011) 4:1873-83. doi: 10.1158/1940-6207.CAPR-11-0218

30. Bowers K, Albanes D, Limburg P, Pietinen P, Taylor PR, Virtamo J, et al. A prospective study of anthropometric and clinical measurements associated with insulin resistance syndrome and colorectal cancer in male smokers. Am J Epidemiol. (2006) 164:652-64. doi: 10.1093/aje/kwj253

31. Mendonca FM, de Sousa FR, Barbosa AL, Martins SC, Araujo RL, Soares, R, et al. Metabolic syndrome and risk of cancer: Which link?[J]. Metabol Clin Exp. (2015) 64:182-9. doi: 10.1016/j.metabol.2014.10.008

32. Guo S. Insulin signaling, resistance, and the metabolic syndrome: insights from mouse models into disease mechanisms. J Endocrinol. (2014) 220:T1-23. doi: 10.1530/JOE-13-0327

33. Arcidiacono B, Iiritano S, Nocera A, Possidente K, Nevolo MT, Ventura V, et al. Insulin resistance and cancer risk: an overview of the pathogenetic mechanisms. Exp Diabetes Res. (2012) 2012:789174. doi: 10.1155/2012/789174

34. Pollak M. Insulin and insulin-like growth factor signalling in neoplasia. Nat Rev Cancer. (2008) 8:915-28. doi: 10.1038/nrc2536

35. Nam SY, Marcus C. Growth hormone and adipocyte function in obesity. Horm Res. (2000) 53(Suppl 1):87-97. doi: 10.1159/000053211

36. Giovannucci E, Harlan DM, Archer MC, Bergenstal RM, Gapstur SM, Habel LA, et al.Diabetes and cancer: a consensus report. Diabetes Care. (2010) 33:1674-85. doi: $10.2337 /$ dc10-0666

37. Aleksandrova K. Influence of obesity and related metabolic alterations on colorectal cancer risk. Curr Nutr Rep. (2013) 2:1-9. doi: $10.1007 / \mathrm{s} 13668-012-0036-9$

38. Grundy SM, Cleeman JI, Daniels SR, Donato KA, Eckel RH, Franklin BA, et al. Diagnosis and management of the metabolic syndrome: an American Heart Association/National heart, lung, and blood institute scientific statement. Circulation. (2005) 112:2735-52. doi: 10.1161/CIRCULATIONAHA.105.169404

Conflict of Interest: The authors declare that the research was conducted in the absence of any commercial or financial relationships that could be construed as a potential conflict of interest.

Copyright (C) 2019 Li, Chen, Wang, Feng, Lyu, Wei, Wen, Chen, Wu, Hang, Dai, Li and He. This is an open-access article distributed under the terms of the Creative Commons Attribution License (CC BY). The use, distribution or reproduction in other forums is permitted, provided the original author(s) and the copyright owner(s) are credited and that the original publication in this journal is cited, in accordance with accepted academic practice. No use, distribution or reproduction is permitted which does not comply with these terms. 